\title{
Echocardiographic evaluation of myocardial strain in patients after transcatheter aortic valve implantation
}

\author{
Tomasz Bochenek ${ }^{1}$, Blazej Kusz ${ }^{1}$, Magdalena Mizia ${ }^{1}$, Michal Lelek ${ }^{1}$, Maciej Turski ${ }^{1}$, Krystian Wita $^{1}$, \\ Andrzej Ochała ${ }^{2}$, Katarzyna Mizia-Stec ${ }^{1}$
}

${ }^{1}$ First Department of Cardiology, Medical University of Silesia, Katowice, Poland

${ }^{2}$ Third Department of Cardiology, Medical University of Silesia, Katowice, Poland

Postep Kardiol Inter 2015; 11, 2 (40): 95-99

DOI: $10.5114 /$ pwki.2015.52281

\begin{abstract}
A bstract
Introduction: Echocardiographic evaluation of regional myocardial function helps to assess the efficacy of therapeutic interventions and to predict the prognosis and clinical outcomes.

Aim: To assess whether myocardial strain can be useful in estimation of left ventricle (LV) function in patients who have undergone transcatheter aortic valve implantation (TAVI).

Material and methods: Twenty-six patients with severe aortic stenosis, who successfully underwent TAVI, were enrolled in the study. Left ventricular peak systolic longitudinal strain (LV PSLS) was obtained before and 1 year after the procedure. Analysis included the potent influence of factors such as sex, LV ejection fraction (LVEF), type of prosthesis implanted or the type of the approach on LV PSLS values.

Results: We observed a significant improvement in LV PSLS values after TAVI $(-10.9 \pm 5.7$ vs. $-13.4 \pm 4.7, p<0.05)$. Men had better improvement in LV PSLS after TAVI, but their starting values were considerably lower (M: $-10.7 \pm 4.5$ before vs. $-13.3 \pm 4.9$ after, $p<0.05$; W: $-11.8 \pm 6.8$ before vs. $-11.9 \pm 5.6$ after, $p=N S$ ). Patients with starting LVEF $\leq 40 \%$ benefited from the procedure (LV PSLS: $-10.3 \pm 6.4$ before vs. $-13.7 \pm 2.9$ after, $p<0.05$ ), but in the group of patients with the higher starting LVEF no significant changes in LV PSLS were observed. We also did not note any differences in LV PSLS depending on type of the prosthesis implemented (Edwards Sapiens/CoreValve). Patients in whom the prostheses were implemented via the femoral approach only presented significant increase in LV PSLS values (before: $-10.4 \pm 6.7$ vs. after: $-13.6 \pm 3.7, p<0.05$ ).

Conclusions: The TAVI results in improvement of LV systolic function according to LV PSLS values. Some factors, especially lower baseline LVEF, are related to increased benefit in LV PSLS after TAVI.
\end{abstract}

Key words: myocardial strain, peak systolic longitudinal strain, aortic valve stenosis, transcatheter aortic valve implantation, transcatheter aortic valve implantation.

\section{Introduction}

The echocardiographic evaluation of the regional myocardial function plays a major role in the diagnosis of various heart diseases - it helps to assess the efficacy of therapeutic interventions and allows one to assess prognosis and clinical outcomes. Despite some well-known advanced diagnostic alternatives for the assessment of ventricular wall motion, echocardiography still remains the safest, easily accessible and consequently the most commonly used method in routine hospital practice. One widely accepted method of assessment of contractile function is the measurement of function either by "eyeball" or, probably more objective but also time consuming, the Simpson method. Nevertheless, the above measurements are affected by the heart rhythm changes and cardiac loading. To improve the process of evaluation of regional myocardial function the echocardiographic strain imaging method has been developed. Strain "itself" is defined as the deformation of an object, normalized to its original shape, and can be calculated as change

in length $(L)$ divided by original length $(L O):\left(\frac{L-L O}{L O}\right)$. It is though a dimensionless quantity which represents the fractional or the percentage change in dimension [1-4]. Strain rate is the speed of deformation. There are several widely used deformation coordinates measured during systole: longitudinal shortening, circumferential shortening and radial thickening. There are studies proving the usefulness of deformation imaging in coronary artery disease management, prediction of the infarction size or cardiotoxicity of cancer therapy [5-8]. Echocardio-

Corresponding author:

Tomasz Bochenek MD, PhD, First Department of Cardiology, Medical University of Silesia, 47 Ziolowa St, 40-635 Katowice, Poland, phone: +48 660689 250, e-mail: tbochun1@gmail.com

Received: 6.03.2015, accepted: 26.03.2015. 
graphic strain imaging could potentially be helpful in the evaluation of left ventricle (LV) function in patients who have undergone transcatheter aortic valve implantation (TAVI), as the aortic stenosis (AS) causes changes in the myocardial strain rate. It is important to underline that the speckle tracking technique is angle independent, thus making the method even more valuable. In patients with severe AS, the relief of LV outflow obstruction leads to obvious changes in the intracardiac hemodynamics. There have already been studies on strain imaging in patients after traditional surgical aortic valve replacement showing promising conclusions. The TAVI is a relatively new therapeutic option for patients with severe symptomatic aortic stenosis who do not qualify for conventional aortic valve replacement. Deformation imaging can help to estimate the long-term clinical outcome of the procedure and to precisely evaluate the LV function - this finally may improve the management and treatment of this group of patients.

\section{Aim}

The aim of the study was to evaluate the impact of TAVI on myocardial longitudinal LV systolic strain in patients with severe degenerative AS. Additionally, we wanted to determine whether sex, baseline LV ejection fraction (LVEF), type of implemented prosthesis or type of the approach influenced myocardial strain values in patients after TAVI.

\section{Material and methods}

The group of 35 patients who successfully underwent TAVI for severe AS (AVA $<0.8 \mathrm{~cm}^{2}$ ) in the years 2012-2014 in our department were primarily enrolled in the study. All patients gave informed consent for participation in the study. From this group 26 patients (M/W: $12 / 14$ ) finally met the criteria, entered and successfully finished the study. Six patients could not come for follow-up due to logistical reasons, and three patients died. The inclusion criteria were: planned TAVI procedure and the achievement of good visibility of the echocardiographic images.

The exclusion criteria included: a bad acoustic window (lack of more than 4 LV segments visualized), non-sinus rhythm, a complicated early post-operative period (i.e. cardiogenic shock, hypovolemic shock or a severe infection), presence of the other significant valvular heart disease that could influence strain assessment, and obesity with body mass index $(\mathrm{BMI})>35 \mathrm{~kg} / \mathrm{m}^{2}$. Patients were treated either percutaneously or transapically, received the Edward Sapien or CoreValve self-expanding prosthesis and underwent complete echocardiographic examination before the procedure and 1 year after.

The study protocol was approved by the local Bioethics Committee. Each patient gave written consent to participate in the study.

\section{Strain analysis}

The assessment of LV strains was done using 2D-STE. Standard 2D grey-scale images from the apical 2-, 3- and 4-chamber views as well as a parasternal short-axis view at the mid-LV level (papillary muscle) were acquired and transferred to a workstation for further offline analysis. The images were taken at a frame rate of 60 to 90 frames/s and were obtained during an end-expiratory breath hold. Strain measurements were done offline using EchoPAC version 6.00 (GE Medical Systems, Milwaukee, WI). The LV peak systolic longitudinal strain (LV PSLS) was averaged from 18 segment measurements from the apical 2-, 3- and 4-chamber views.

The technique for strain measurement required the manual outlining of the LV endocardial contour, after which the system automatically generated the myocardial contour in the end-systolic frame. The myocardial tracking was verified manually and, if necessary, a strain analysis was done by dividing each LV image into six segments per view. The system generated the curves for each LV segment. The LV PSLS was the maximal negative strain value during the ejection phase with the beginning of the QRS complex and the aortic valve close time as the reference points. Segments with poor visualization were excluded from further analysis. Patients in whom more than four segments could not be analyzed were excluded. Among many others, the following parameters were calculated: LVEF, peak aortic velocity, peak and mean systolic pressure gradient.

The LV PSLS was measured for all of the strain parameters for all of the analyzed segments and averaged to derive a mean value that was used for the analysis. All strain measurements were done by one experienced observer. Ten studies were reanalyzed by the same observer in order to assess intra-observer variability. The intra-observer variability for LV PSLS was $5 \%$.

\section{Statistical analysis}

Statistical analysis was performed using Statistica version 10. Full analysis of the descriptive statistical parameters was performed and the most important of them were particularized in the study. All of the text and table results are expressed as means \pm standard deviation (SD) or a number (percentage).

Analysis included the comparison of LV PSLS values before and after TAVI for the group of all patients as well as depending on sex, LVEF, type of the prosthesis and type of the approach. The type of distribution was verified using the Shapiro-Wilk test. In the case of normally distributed variables, Student's $t$ test for unpaired samples was used, while the Mann-Whitney $U$ test was used in the case of non-normally distributed parameters. Qualitative variables were compared using the $\chi^{2}$ test with Yates correction. The Spearman rank-order test or Pearson correlations were used to determine the rela- 
tionship between variables. A $p$ value of $<0.05$ was considered statistically significant.

\section{Results}

\section{Clinical data}

Our study finally included 26 patients (14 female, 12 male; mean age: $77 \pm 8$ years) who were disqualified from a traditional surgical approach and qualified for TAVI according to high EuroSCORE values and following a local heart team decision. Mean EuroSCORE II was $24.18 \%$ and STS $11.43 \%$.

All the patients were symptomatic: NYHA II: 11 (42\%) patients, NYHA III: 15 (58\%) patients. Co-morbidities in the patients were as follows: 23 (86\%) patients suffered from systemic hypertension, 17 (65\%) had previously diagnosed coronary artery diseases, while 5 (19\%) reported previous coronary artery bypass grafting and 14 (54\%) underwent previous percutaneous coronary intervention; 14 (54\%) patients had general atherosclerosis, including significant narrowing of carotid arteries in 9 (35\%) cases. In 9 (35\%) cases there were reports of prior myocardial infarction.

\section{Procedure data}

The TAVI procedures were performed using a transapical $(11 ; 42 \%)$ or transfemoral $(15 ; 58 \%)$ approach. Twelve (46\%) patients received a CoreValve prosthesis, while 14 (54\%) patients had an Edwards Sapien Valve prosthesis implanted. No clinical complications were observed in the early post-procedural period.

\section{Follow-up data}

Clinical analysis performed 1 year after TAVI revealed significant clinical improvement. None of the patients was hospitalized during this period. The majority of patients presented less severe heart failure symptoms. An improvement on the NYHA scale was reported: NYHA II in 16 patients (62\%), NYHA III in 10 patients (38\%).

\section{TTE 1 year after TAVI}

We observed a significant reduction in maximal (89.1 $\pm 22.4 \mathrm{~mm} \mathrm{Hg}$ vs. $24.1 \pm 11.2 \mathrm{~mm} \mathrm{Hg}, p<0.001$ ) as well as

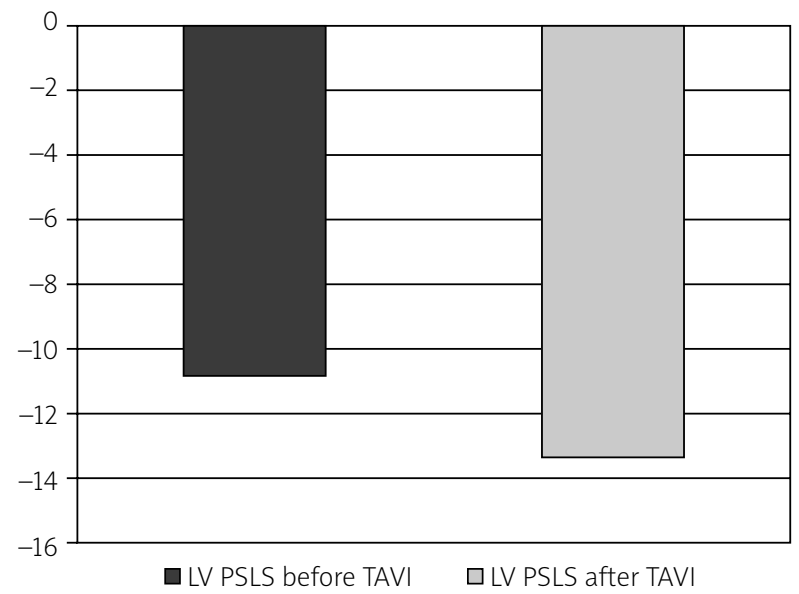

Figure 1. Left ventricle peak systolic longitudinal strain before and after transcatheter aortic valve implantation $(p<0.05)$

in mean transaortic gradient $(53.5 \pm 15.9 \mathrm{~mm} \mathrm{Hg}$ vs. 10.9 $\pm 9.3 \mathrm{~mm} \mathrm{Hg}, p<0.001)$ and in maximum aortic jet velocity $(4.7 \pm 0.7 \mathrm{~m} / \mathrm{s}$ vs. $2.3 \pm 0.6 \mathrm{~m} / \mathrm{s}, p<0.05)$ in patients after TAVI.

There were no significant differences in LVEF before and 1 year after TAVI $(47.3 \pm 11.0 \%$ vs. $50.0 \pm 8.3 \%$, $p=N S)$. The LV PSLS significantly increased after the surgery $(-10.9 \pm 5.7$ vs. $-13.4 \pm 4.7, p<0.05)$ (Figure 1$)$.

\section{Clinical factors and potent influence on LV PSLS (Table I) \\ Gender}

Mean LV PSLS values both before and after TAVI were slightly worse in 12 (46\%) men than in 14 (54\%) women with significant improvement after the procedure in men (before: $-10.7 \pm 4.5$, after: $-13.3 \pm 4.9, p<0.05$ ).

\section{LVEF}

Mean LV PSLS values in 12 (46\%) patients with LVEF $\leq 40 \%$ were insignificantly lower compared to those observed in 14 (54\%) patients with LVEF > 40\% (-10.3 \pm 6.4 vs. $-12.2 \pm 4.9, p=N S)$. These values increased greatly

Table I. Clinical factors and potent influence on left ventricular peak systolic longitudinal strain

\begin{tabular}{|c|c|c|c|c|c|c|c|c|c|c|c|c|}
\hline \multirow[t]{2}{*}{ Variable } & \multicolumn{3}{|c|}{ LV PSLS } & \multicolumn{3}{|c|}{ LVEF } & \multicolumn{3}{|c|}{ Type of prosthesis } & \multicolumn{3}{|c|}{ Type of approach } \\
\hline & $\begin{array}{c}\text { Male } \\
(n=12) \\
\text { Mean } \\
\pm \text { SD }\end{array}$ & $\begin{array}{l}\text { Female } \\
(n=14) \\
\text { Mean } \\
\pm \text { SD }\end{array}$ & $\begin{array}{l}\text { Value } \\
\text { of } p\end{array}$ & $\begin{array}{c}\leq 40 \% \\
(n=12) \\
\text { Mean } \\
\pm \text { SD }\end{array}$ & $\begin{array}{c}>40 \% \\
(n=14) \\
\text { Mean } \\
\pm \text { SD }\end{array}$ & $\begin{array}{l}\text { Value } \\
\text { of } p\end{array}$ & $\begin{array}{c}\text { CoreValve } \\
(n=12) \\
\text { Mean } \\
\pm \text { SD }\end{array}$ & $\begin{array}{c}\text { Edwards } \\
\text { Sapiens } \\
(n=14) \\
\text { Mean } \pm \text { SD }\end{array}$ & $\begin{array}{l}\text { Value } \\
\text { of } p\end{array}$ & $\begin{array}{c}\text { Transapical } \\
\begin{array}{c}(n=11) \\
\text { Mean } \\
\pm \text { SD }\end{array}\end{array}$ & $\begin{array}{c}\text { Transfemoral } \\
(n=15) \\
\text { Mean } \\
\pm \text { SD }\end{array}$ & $\begin{array}{l}\text { Value } \\
\text { of } p\end{array}$ \\
\hline Before & $\begin{array}{l}-10.7 \\
\pm 4.5 \\
\end{array}$ & $\begin{array}{l}-11.8 \\
\pm 6.8 \\
\end{array}$ & NS & $\begin{array}{c}-10.3 \\
\pm 6.4 \\
\end{array}$ & $\begin{array}{l}-12.2 \\
\pm 4.9 \\
\end{array}$ & NS & $-11.9 \pm 6.0$ & $-10.6 \pm 5.4$ & NS & $-11.9 \pm 4.1$ & $-10.4 \pm 6.7$ & NS \\
\hline $\begin{array}{l}\text { One year } \\
\text { after }\end{array}$ & $\begin{array}{l}-13.3 \\
\pm 4.9\end{array}$ & $\begin{array}{c}-11.9 \\
\pm 5.6\end{array}$ & NS & $\begin{array}{l}-13.7 \\
\pm 2.9\end{array}$ & $\begin{array}{l}-11.5 \\
\pm 6.7\end{array}$ & $\begin{array}{c}< \\
0.05\end{array}$ & $-13.1 \pm 4.1$ & $-12.0 \pm 6.3$ & NS & $-11.1 \pm 6.0$ & $-13.6 \pm 3.7$ & NS \\
\hline $\begin{array}{l}\text { Value of } \\
p \text { : before } \\
\text { vs. after }\end{array}$ & $<0.05$ & NS & & $<0.05$ & NS & & NS & NS & & NS & $<0.05$ & \\
\hline
\end{tabular}


after TAVI in the first group $(-13.7 \pm 2.9, p<0.05)$ but decreased insignificantly in patients with the starting LVEF $>40 \%(-11.5 \pm 6.7, p=\mathrm{NS})$.

We observed a weak negative correlation between LVEF and LV PSLS values ( $r=-0.3, p=N S)$.

\section{Type of prosthesis implanted}

There were no significant differences in either baseline or post-TAVI mean LV PSLS values in patients qualified for a CoreValve prosthesis (before: $-11.9 \pm 6.0$ vs. after: $-13.1 \pm 4.1, p=N S$ ) or an Edward Sapiens prosthesis (before: $-10.6 \pm 5.4$ vs. after: $-12.0 \pm 6.3, p<0.05$ ).

\section{Type of approach}

Patients who received a prosthesis via a transapical approach had insignificantly greater starting values of LV PSLS $(-11.9 \pm 4.1)$ than those with transfemoral access $(-10.4 \pm 6.7)$, but only the second (transfemoral) group significantly benefited from the procedure $(-13.6 \pm 3.7$, $p<0.05)$ while in the first one (transapical) LV PSLS values decreased insignificantly.

\section{Discussion}

The TAVI has already been a well-studied approach, especially in patients with high risk of traditional surgical valve replacement. It appeared to be a reasonable option especially in elderly patients [8]. Dr Alain Cribier pioneered the first TAVI procedure in 2002 - since then the new method has emerged with high speed worldwide, providing a great option for treatment of patients, mainly those disqualified from traditional surgery. Nine hundred procedures were done in Poland from November 2008 to June 2013 [9]. Following the guidelines, TAVI should be undertaken with a heart team approach, which was a criterion fulfilled in all of our cases.

The TAVI influences ventricular function. Despite the sometimes described depression of both systolic and diastolic ventricular function within the first 24 postoperative hours [10], significant improvement of ventricular function is usually observed in longer follow-up. Moreover, there are already new studies showing that TAVI results in geometric changes of LV and improves left heart function, as assessed by 2DSTE. To our knowledge, only a few studies concerning this subject have been published worldwide, yet no study has been conducted on this subject in Poland.

In our study we observed an improvement in mean LV PSLS in patients 1 year after TAVI.

It should be noted that there were no significant differences in LVEF. Thus the novel method used in the study seems to be a more sensitive tool in the assessment of LV function.

Additionally, we would like to underline that this improvement was clearly more evident in patients with starting $\mathrm{EF} \leq 40 \%$.
In 2012, Schueler's group [11] also reported that significant improvement of left ventricular global longitudinal strain (LVGLS) was observed 6 months after TAVI, and it was observable mainly in patients with impaired LV function. The results of the study are also consistent with the results from the group of D'Andrea et al. recently published in the journal Echocardiography [12]. Another group has also presented results indicating that TAVI improves global and regional LV mechanics within 12 months. This may be explained by a simple explanation: TAVI, like other approaches of valve surgery, reduces ventricular overload, and thus influences geometrics.

Grabskaya et al. [13] claim that mainly longitudinal mechanics respond to unloading of the left ventricle after TAVI for severe aortic stenosis while circumferential deformation remains substantially unchanged. Recent studies comparing longitudinal and circumferential function have shown that longitudinal function deteriorates early under cardiac pathologic conditions before the onset of clinical symptoms and reduction in global ventricular function, whereas circumferential function might remain relatively preserved to compensate for cardiac function when longitudinal function starts to become dysfunctional [14].

The potential role of the influence of sex on strain values is interesting. Our study demonstrated that mean LV PSLS values both before and after TAVI were slightly worse in men than in women, but improvement was slightly more noticeable in men. In the Framingham Heart Study, in a healthy group of people in multivariable analyses women had greater longitudinal strain, greater transverse strain, and greater circumferential strain compared with men. Separate reference limits for measures of left ventricular strain and synchrony were observed for both sexes [15]. Also Kocabay et al. [16] found that women have more negative longitudinal strain.

Our study demonstrated that mean LV PSLS values in patients with LVEF $\leq 40 \%$ were lower than those observed in patients with LVEF > $40 \%$. These values increased after TAVI in the first group but decreased insignificantly in patients with starting LVEF $>40 \%$. This conclusion is relatively obvious. According to a recent a study, TAVI restores LV function toward more physiologic myocardial mechanics in both normal- and depressed-LVEF groups; however, patients with lower systolic function derive the most benefit in terms of longitudinal reverse remodeling [17]. Also results from the group of D'Ascenzi et al. [18] indicated that significant improvements of LVEF and LVGLS can be observed in patients undergoing TAVI mainly in those with basically impaired LVEF.

As to type of prosthesis, values generally improved in both subgroups. In our study we observed that patients qualified for a CoreValve prosthesis had better starting LV PSLS values.

Patients from the transapical group had greater starting values of LV PSLS than those in the transfemoral 
group. We observed a significant improvement in LV PSLS in the group that received a prosthesis via the transfemoral approach, but these values decreased insignificantly in the group where pericardial access was used. One may hypothesize that the transapical approach may induce more injury to the muscle itself thus it may potentially lead to worse improvement in strains especially focusing on apical regions. In a study published in JACC [19] the conclusion was that TAVI was systematically associated with some degree of myocardial injury, with the transapical approach determining a higher increase in biomarkers of myocardial injury. According to the authors, a greater degree of myocardial injury was associated with less improvement in LVEF, which is consistent with our data. Moreover, it was documented that a cardiac biomarker increase after transcatheter aortic valve replacement (TAVR) was common and more frequent among transapical access patients [20].

We are aware that lack of rotational and circumferential strain is a limitation. Nevertheless, the majority of studies published find longitudinal strain as a key deformation parameter.

Our study is based on novel technologies but has some limits, which we are well aware of. The relatively low number of TAVI performed every year in Poland as well as in our center influenced the number of patients finally included in the study. The number of patients could have an impact on statistical data and constitutes a limitation for multivariate analysis.

In our study echocardiographic strain imaging was performed by a highly trained operator; however, high intra-observer variability of the technique itself is a limitation. Another interesting point lacking in the study would be validation of our data against magnetic resonance strain imaging data, which could significantly enhance assessment of deformations.

\section{Conclusions}

The TAVI results in improvement of longitudinal myocardial strain values, and thus has an impact on geometrical deformation of the left ventricle. The novel type of imaging seems useful in evaluation, but further research is needed to standardize and objectify the method. It seems important to consider the role of clinical factors influencing LV global systolic function after TAVI, but further studies are needed to confirm this hypothesis.

\section{Conflict of interest}

The authors declare no conflict of interest.

\section{References}

1. John G, Tanaka H. Echocardiographic assessment of myocardial strain. J Am Coll Cardiol 2011; 58: 1401-13.

2. Mirsky I, Parmley WW. Assessment of passive elastic stiffness for isolated heart muscle and the intact heart. Circ Res 1973; 33: 233-43.
3. D'hooge J, Heimdal A, Jamal F, et al. Regional strain and strain rate measurements by cardiac ultrasound: principles, implementation and limitations. Eur J Echocardiogr 2000; 1: 154-70.

4. Teske AJ, De Boeck BW, Melman PG, et al. Echocardiographic quantification of myocardial function using tissue deformation imaging, a guide to image acquisition and analysis using tissue Doppler and speckle tracking. Cardiovasc Ultrasound 2007; 5: 27.

5. Hoit BD. Strain and strain rate echocardiography and coronary artery disease. Circ Cardiovasc Imaging 2011; 4: 179-90.

6. Miyasaka Y, Haiden M, Kamihata $H$, et al. Usefulness of strain rate imaging in detecting ischemic myocardium during dobutamine stress. Int J Cardiol 2005; 102: 225-31.

7. Thavendiranathan P, Poulin F, Lim KD, et al. Use of myocardial strain imaging by echocardiography for the early detection of cardiotoxicity in patients during and after cancer chemotherapy: a systematic review. J Am Coll Cardiol 2014; 6: 2751-68.

8. Gilard M, Eltchaninoff $\mathrm{H}$, lung B, et al. FRANCE 2 Investigators. Registry of transcatheter aortic-valve implantation in high-risk patients. N Engl J Med 2012; 366: 1705-15.

9. Dąbrowski M, Witkowski A. Stroke following transcatheter aortic valve implantation. Is neuroprotection justified? Postep Kardiol Inter 2013; 9: 376-82.

10. Dworakowski R, Wendler O, Bhan A, et al. Successful transcatheter aortic valve implantation (TAVI) is associated with transient left ventricular dysfunction. Heart 2012; 98: 1641-6.

11. Schueler R, Sinning JM, Momcilovic D, et al. Three-dimensional speckle-tracking analysis of left ventricular function after transcatheter aortic valve implantation. J Am Soc Echocardiogr 2012; 25: 827-34.

12. D'Andrea A, Padalino R, Cocchia R, et al. Effects of transcatheter aortic valve implantation on left ventricular and left atrial morphology and function. Echocardiography 2014 Oct 17. doi: 10.1111/echo. 12808

13. Grabskaya E, Becker M, Altiok E, et al. Impact of transcutaneous aortic valve implantation on myocardial deformation. Echocardiography 2011; 28: 397-401.

14. Hung CL, Verma $A$, Uno $H$, et al. VALIANT investigators. Longitudinal and circumferential strain rate, left ventricular remodeling, and prognosis after myocardial infarction. J Am Coll Cardiol 2010; 56: 1812-22.

15. Cheng S, Larson MG, McCabe EL, et al. Age- and sex-based reference limits and clinical correlates of myocardial strain and synchrony: the Framingham Heart Study. Circ Cardiovasc Imaging 2013; 6: 692-9.

16. Kocabay G, Muraru D, Peluso D, et al. Normal left ventricular mechanics by two-dimensional speckle-tracking echocardiography. Reference values in healthy adults. Rev Esp Cardiol (Engl Ed) 2014; 67: 651-8.

17. Poulin F, Carasso S, Horlick EM, et al. Recovery of left ventricular mechanics after transcatheter aortic valve implantation: effects of baseline ventricular function and postprocedural aortic regurgitation J Am Soc Echocardiogr 2014; 27: 1133-42.

18. D’Ascenzi F, Cameli M, ladanza A, et al. Improvement of left ventricular longitudinal systolic function after transcatheter aortic valve implantation: a speckle-tracking prospective study. Int J Cardiovasc Imaging 2013; 29: 1007-15.

19. Rodés-Cabau J, Gutiérrez M, Bagur R, et al. Incidence, predictive factors, and prognostic value of myocardial injury following uncomplicated transcatheter aortic valve implantation. JACC 2011; 57: 1988-99.

20. Barbash IM, Dvir D, Ben-Dor I. et al. Prevalence and effect of myocardial injury after transcatheter aortic valve replacement. Am J Cardiol 2013; 111: 1337-43. 